\title{
Chemoselective Syntheses of $\gamma$-Butyrolactams using Vinyl Sulfilimines and Dichloroketene
}

\author{
Joseph P. Marino* and Nanfei Zou \\ Department of Chemistry and Biochemistry, University of Notre Dame, \\ Notre Dame, IN 46556 \\ Email Address: marino.12@nd.edu
}

\section{Supporting Information}

\section{General}

All reactions were conducted in flame dried glassware under nitrogen. All solvents were purified before use. $\mathrm{THF}$ and $\mathrm{Et}_{2} \mathrm{O}$ were dried by distillation from sodium benzophenone ketyl. $\mathrm{CH}_{2} \mathrm{Cl}_{2}, \mathrm{DMF}, \mathrm{CH}_{3} \mathrm{CN}$ were dried by distillation from $\mathrm{CaH}_{2}$. Methanol was distilled from magnesium turnings. Toluene was dried by distillation from sodium. All other commercially available reagents and solvents were used as received.

${ }^{1} \mathrm{H}$ NMR data were recorded at $500 \mathrm{MHz}$ or $400 \mathrm{MHz}$ using a Varian INOVA 500 or a Bruker Avance 400 instrument respectively. ${ }^{1} \mathrm{H}$ NMR chemical shifts are reported as delta $(\delta)$ units in parts per million (ppm) relative to residual $\mathrm{CHCl}_{3}(7.26 \mathrm{ppm}) .{ }^{13} \mathrm{C} \mathrm{NMR}$ data were recorded at $125 \mathrm{MHz}$ or $100 \mathrm{MHz}$ using a Varian INOVA 500 or a Bruker Avance 400 instrument respectively. Coupling constants $(J)$ were reported in $\operatorname{Hertz}(\mathrm{Hz})$. ${ }^{13} \mathrm{C}$ NMR chemical shifts were reported as delta $(\delta)$ units in parts per million (ppm) relative to the central line of $\mathrm{CDCl}_{3}(77.0 \mathrm{ppm})$. Infrared spectra were recorded using a Perkin Elmer Paragon 1000 FT-IR and were reported in wavelength numbers $\left(\mathrm{cm}^{-1}\right)$. High resolution mass spectroscopy was performed on a JOEL-AX505HA mass spectrometer at the University of Notre Dame Mass Spectrometry Facility.

Reactions were monitored by thin-layer chromatography on $0.25 \mathrm{~mm}$ E. Merck silica gel 60 plates $\left(\mathrm{F}_{254}\right)$ using UV light and ammonium molybdate-cerium ammonium nitrate as visualizing agents. Column chromatographic purification were performed using

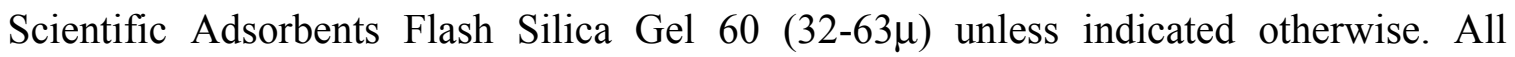


compounds purified by chromatography were sufficiently pure for use in further experiments, unless indicated otherwise.

\section{General Lactamization Procedure (synthesis of lactam 9 of Table 2 as an example)}

To a dry flask equipped with magnetic stir bar, temperature probe and a condenser was added zinc powder (500 mg, $7.35 \mathrm{mmol})$, copper (I) chloride (728 mg, $7.35 \mathrm{mmol}$ ) and anhydrous THF $4 \mathrm{~mL}$. Heat the reaction mixture to reflux under nitrogen and keep it over 1.5 hours. Cool the reaction to $-30^{\circ} \mathrm{C}$. Transfer sulfilimine 8 (135 $\left.\mathrm{mg}, 0.37 \mathrm{mmol}\right)$ in THF $3 \mathrm{~mL}$ to the zinc-copper mixture via cannula. While keeping the reaction at $-30^{\circ} \mathrm{C}$, charge trichloroacetyl chloride $(205 \mu \mathrm{L}, 1.84 \mathrm{mmol})$ to the reaction via a syringe pump in 0.5 hour. Sample the reaction for TLC monitoring. Upon the disappearance of sulfilimine, the reaction mixture was filtered through a celite pad into sodium bicarbonate solution 5 $\mathrm{mL}$. Extract the filtrate solution with ethyl acetate $(2 \times 10 \mathrm{~mL})$. Wash the combined organic layers with brine $5 \mathrm{~mL}$. Dry the organic solution with magnesium sulfate. Filter. Remove solvent in vacuo to give crude product lactam 9. Purify the crude product via silica gel chromatography with ethyl acetate / hexanes $(5: 95)$ to give $145 \mathrm{mg}(82 \%)$ of lactam 9.

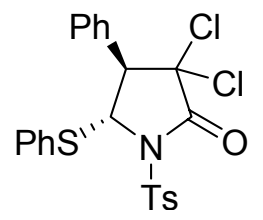

\section{Lactam 6a}

\section{3,3-Dichloro-4-phenyl-5-phenylsulfanyl-1-(toluene-4-sulfonyl)-pyrrolidin-2-one} (Table 1, entry a)

${ }^{1} \mathrm{H}$ NMR $\left(500 \mathrm{MHz} \mathrm{CDCl}_{3}\right) \delta 8.20(\mathrm{~d}, J=8.4 \mathrm{~Hz}, 2 \mathrm{H}), 7.48-7.25(\mathrm{~m}, 10 \mathrm{H}), 7.08(\mathrm{~m}, 2$ $\mathrm{H}), 5.70(\mathrm{~d}, J=8.3 \mathrm{~Hz}, 1 \mathrm{H}), 3.57(\mathrm{~d}, J=8.3 \mathrm{~Hz}, 1 \mathrm{H}), 2.52(\mathrm{~s}, 3 \mathrm{H}) ;{ }^{13} \mathrm{C}$ NMR $(125$

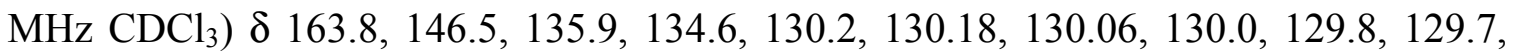
129.6, 129.0, 128.9, 83.4, 67.4, 59.6, 22.1; IR (thin film) 2924, 1760, 1372, 1226, 1187 , $1169,1085,975,873 \mathrm{~cm}^{-1}$; HRMS (FAB) calculated for $\mathrm{C}_{23} \mathrm{H}_{20} \mathrm{Cl}_{2} \mathrm{NO}_{3} \mathrm{~S}_{2}, 492.0262 \mathrm{~m} / \mathrm{z}$ $(\mathrm{M}+\mathrm{H})^{+}$; observed 492.0252 . 


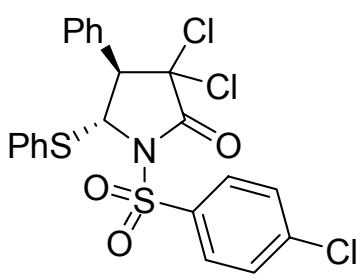

Lactam 6b

3,3-Dichloro-1-(4-chloro-benzenesulfonyl)-4-phenyl-5-phenylsulfanyl-pyrrolidin-2one (Table 1, entry b)

${ }^{1} \mathrm{H}$ NMR $\left(500 \mathrm{MHz} \mathrm{CDCl}_{3}\right) \delta 8.27(\mathrm{~d}, J=8.7 \mathrm{~Hz}, 2 \mathrm{H}), 7.64(\mathrm{~d}, J=8.6 \mathrm{~Hz}, 2 \mathrm{H}), 7.50$ $7.26(\mathrm{~m}, 8 \mathrm{H}), 7.07$ (d, $J=7.2 \mathrm{~Hz}, 2 \mathrm{H}), 5.71(\mathrm{~d}, J=8.3 \mathrm{~Hz}, 1 \mathrm{H}), 3.61(\mathrm{~d}, J=8.4 \mathrm{~Hz}, 1$ $\mathrm{H}) ;{ }^{13} \mathrm{C} \mathrm{NMR}\left(125 \mathrm{MHz} \mathrm{CDCl}_{3}\right) \delta 163.7,142.0,135.9,135.6,130.9,130.2,130.1,129.8$, 129.74, 129.70, 129.68, 129.0, 128.8, 83.1, 67.6, 59.8; IR (thin film) 2923, 1766, 1584, 1476, 1380, 1230, 1185, 1174, 1093, 976, 759, $693 \mathrm{~cm}^{-1}$; HRMS (FAB) calculated for $\mathrm{C}_{22} \mathrm{H}_{17} \mathrm{Cl}_{3} \mathrm{NO}_{3} \mathrm{~S}_{2}, 511.9715 \mathrm{~m} / \mathrm{z}(\mathrm{M}+\mathrm{H})^{+}$; observed 511.9726.<smiles>O=S(=O)(N=C1O[C@H]([SnH]c2ccccc2)C([PH+]([O-])c2ccccc2)C1(Cl)Cl)c1ccc(Cl)cc1</smiles>

Lactone Imine $7 b$

\section{4-Chloro-N-(3,3-dichloro-4-phenyl-5-phenylsulfanyl-dihydro-furan-2-ylidene)-} benzenesulfonamide (Table 1 , entry $b$ )

${ }^{1} \mathrm{H}$ NMR $\left(500 \mathrm{MHz} \mathrm{CDCl}_{3}\right) \delta 8.02(\mathrm{~d}, J=8.6 \mathrm{~Hz}, 2 \mathrm{H}), 7.54(\mathrm{~d}, J=8.6 \mathrm{~Hz}, 2 \mathrm{H}), 7.51-$ 7.43 (m, $6 \mathrm{H}), 7.32-7.29$ (m, $4 \mathrm{H}), 6.19$ (d, $J=10.5 \mathrm{~Hz}, 1 \mathrm{H}), 3.72$ (d, $J=10.5 \mathrm{~Hz}, 1 \mathrm{H})$; ${ }^{13} \mathrm{C}$ NMR $\left(125 \mathrm{MHz} \mathrm{CDCl}_{3}\right) \delta 165.3,140.0,138.8,135.1,130.4,130.0,129.9,129.8$, 129.4, 129.3, 129.1, 128.4, 128.1, 93.6, 84.1, 61.3; IR (thin film) 3064, 2923, 1665, 1340, $1167,1092,770 \mathrm{~cm}^{-1}$; HRMS (FAB) calculated for $\mathrm{C}_{22} \mathrm{H}_{17} \mathrm{Cl}_{3} \mathrm{NO}_{3} \mathrm{~S}_{2}, 511.9715 \mathrm{~m} / z(\mathrm{M}+$ H) ${ }^{+}$; observed 511.9706 . 


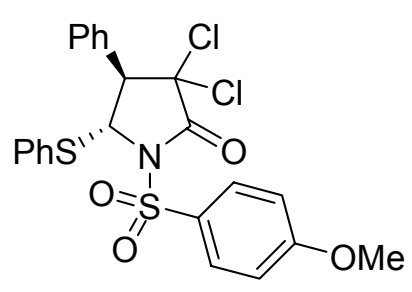

\section{Lactam 6c}

\section{3,3-Dichloro-1-(4-methoxy-benzenesulfonyl)-4-phenyl-5-phenylsulfanyl-pyrrolidin-}

\section{2-one (Table 1, entry c)}

${ }^{1} \mathrm{H}$ NMR $\left(500 \mathrm{MHz} \mathrm{CDCl}_{3}\right) \delta 8.25(\mathrm{~d}, J=9.0 \mathrm{~Hz}, 2 \mathrm{H}), 7.48-7.26(\mathrm{~m}, 8 \mathrm{H}), 7.12(\mathrm{~d}, J=$ $7.9 \mathrm{~Hz}, 2 \mathrm{H}), 7.10$ (d, $J=9.1 \mathrm{~Hz}, 2 \mathrm{H}), 5.69$ (d, $J=8.3 \mathrm{~Hz}, 1 \mathrm{H}), 3.94$ (s, $3 \mathrm{H}), 3.59$ (d, $J$ $=8.2 \mathrm{~Hz}, 1 \mathrm{H}) ;{ }^{13} \mathrm{C}$ NMR $\left(125 \mathrm{MHz} \mathrm{CDCl}_{3}\right) \delta 164.9,163.7,135.8,134.7,132.0,130.08$, $130.05,129.65,129.60,128.9,128.6,127.2,83.1,67.3,59.5,56.0$; IR (thin film) 3063, 2945, 1763, 1594, 1498, 1374, 1267, 1191, 1167, 1089, 1025, 976, 835, 805, 756, 738, $693 \mathrm{~cm}^{-1}$; HRMS (FAB) calculated for $\mathrm{C}_{23} \mathrm{H}_{20} \mathrm{Cl}_{2} \mathrm{NO}_{4} \mathrm{~S}_{2}, 508.0211 \mathrm{~m} / z(\mathrm{M}+\mathrm{H})^{+}$; observed 508.0237.<smiles>COc1ccc(S(=O)(=O)N=C2O[C@@H](c3ccccc3)C([PH+](=O)[O-])[C@H]2c2ccccc2)cc1</smiles>

\section{Lactone Imine 7c}

\section{N-(3,3-Dichloro-4-phenyl-5-phenylsulfanyl-dihydro-furan-2-ylidene)-4-methoxy-} benzenesulfonamide (Table 1 , entry c)

${ }^{1} \mathrm{H}$ NMR $\left(500 \mathrm{MHz} \mathrm{CDCl}_{3}\right) \delta 8.02(\mathrm{~d}, J=8.8 \mathrm{~Hz}, 2 \mathrm{H}), 7.52-7.42(\mathrm{~m}, 8 \mathrm{H}), 7.30(\mathrm{~d}, J=$ $8.5 \mathrm{~Hz}, 2 \mathrm{H}), 7.03$ (d, $J=8.8 \mathrm{~Hz}, 2 \mathrm{H}), 6.15$ (d, $J=10.5 \mathrm{~Hz}, 1 \mathrm{H}), 3.91$ (s, $3 \mathrm{H}), 3.69$ (d, $J$ $=10.5 \mathrm{~Hz}, 1 \mathrm{H}) ;{ }^{13} \mathrm{C} \mathrm{NMR}\left(125 \mathrm{MHz} \mathrm{CDCl}_{3}\right) \delta 164.4,163.6,135.0,131.9,130.3,130.2$, $130.2,130.0,129.9,129.1,128.6,128.3,114.2,93.1,84.2,61.3,55.8$; IR (thin film) 3064, 1666, 1596, 1498, 1337, 1262, 1159, 1093, 1022, 806, 743, $692 \mathrm{~cm}^{-1}$; HRMS (FAB) calculated for $\mathrm{C}_{23} \mathrm{H}_{20} \mathrm{Cl}_{2} \mathrm{NO}_{4} \mathrm{~S}_{2}, 508.0211 \mathrm{~m} / z(\mathrm{M}+\mathrm{H})^{+}$; observed 508.0244. 


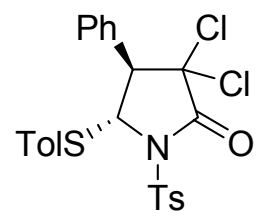

\section{Lactam $6 \mathbf{f}$}

\section{3,3-Dichloro-4-phenyl-1-(toluene-4-sulfonyl)-5-p-tolylsulfanyl-pyrrolidin-2-one}

\section{(Table 1, entry f)}

${ }^{1} \mathrm{H}$ NMR (500 MHz $\left.\mathrm{CDCl}_{3}\right) \delta 8.21(\mathrm{~d}, J=8.4 \mathrm{~Hz}, 2 \mathrm{H}), 7.47-7.44(\mathrm{~m}, 9 \mathrm{H})$, 7.29-7.27 (m, $2 \mathrm{H}), 7.06(\mathrm{~d}, J=8.0 \mathrm{~Hz}, 2 \mathrm{H})$, ), $6.91(\mathrm{~d}, J=8.1 \mathrm{~Hz}, 2 \mathrm{H}), 5.67$ (d, $J=8.5 \mathrm{~Hz}, 1 \mathrm{H}), 3.55$ $(\mathrm{d}, J=8.5 \mathrm{~Hz}, 1 \mathrm{H}), 2.52(\mathrm{~s}, 3 \mathrm{H}), 2.35(\mathrm{~s}, 3 \mathrm{H}) ;{ }^{13} \mathrm{C} \mathrm{NMR}\left(125 \mathrm{MHz} \mathrm{CDCl}_{3}\right) \delta 163.7$, 146.4, 140.6, 136.0, 134.6, 130.4, 130.2, 129.94, 129.85, 129.6, 129.5, 128.9, 124.8, 83.5, 67.2, 59.1, 22.0, 21.4; IR (thin film) 3035, 2923, 1765, 1596, 1493, 1456, 1374, 1310, 1232, 1174, 1085, 977, 910, 875, 814, 764, 702, 662, $567 \mathrm{~cm}^{-1}$; HRMS (FAB) calculated for $\mathrm{C}_{24} \mathrm{H}_{22} \mathrm{Cl}_{2} \mathrm{NO}_{3} \mathrm{~S}_{2}, 506.0418 \mathrm{~m} / z(\mathrm{M}+\mathrm{H})^{+}$; observed 506.0403.

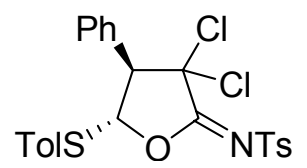

\section{Lactone Imine $7 f$}

N-(3,3-Dichloro-4-phenyl-5-p-tolylsulfanyl-dihydro-furan-2-ylidene)-4-methylbenzenesulfonamide (Table 1 , entry $f$ )

${ }^{1} \mathrm{H}$ NMR (500 MHz $\left.\mathrm{CDCl}_{3}\right) \delta$ 7.98-7.96 (m, $\left.2 \mathrm{H}\right)$, 7.49-7.27 (m, $\left.9 \mathrm{H}\right), 7.21(\mathrm{~d}, J=8.0 \mathrm{~Hz}$, $2 \mathrm{H}), 6.10(\mathrm{~d}, J=10.5 \mathrm{~Hz}, 1 \mathrm{H}), 3.63$ (d, $J=10.5 \mathrm{~Hz}, 1 \mathrm{H}), 2.47$ (s, $3 \mathrm{H}), 2.41(\mathrm{~s}, 3 \mathrm{H})$;

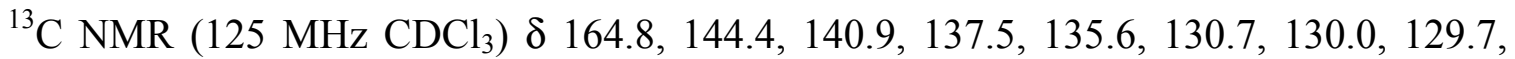
129.1, 128.7, 128.0, 124.2, 93.3, 84.4, 61.2, 22.0, 21.6; IR (thin film) 3035, 2923, 1668, 1597, 1495, 1456, 1337, 1239, 1164, 1091, 1018, 911, 853, 815, 745, 698, 654, 611, 567 $\mathrm{cm}^{-1}$; HRMS (FAB) calculated for $\mathrm{C}_{24} \mathrm{H}_{22} \mathrm{Cl}_{2} \mathrm{NO}_{3} \mathrm{~S}_{2}, 506.0418 \mathrm{~m} / z(\mathrm{M}+\mathrm{H})^{+}$; observed 506.0433 . 


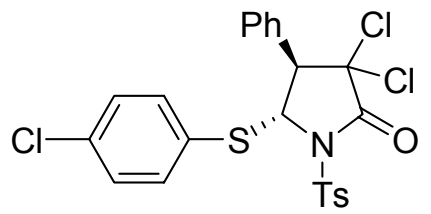

Lactam 6g

3,3-Dichloro-5-(4-chloro-phenylsulfanyl)-4-phenyl-1-(toluene-4-sulfonyl)-pyrrolidin2-one (Table 1, entry g)

${ }^{1} \mathrm{H}$ NMR $\left(500 \mathrm{MHz}_{\mathrm{CDCl}}\right) \delta 8.17(\mathrm{~d}, J=8.3 \mathrm{~Hz}, 2 \mathrm{H})$, 7.49-7.44 (m, $\left.5 \mathrm{H}\right)$, 7.28-7.24 (m, $4 \mathrm{H}), 7.04(\mathrm{~d}, J=8.6 \mathrm{~Hz}, 2 \mathrm{H}), 5.65(\mathrm{~d}, J=8.3 \mathrm{~Hz}, 1 \mathrm{H}), 3.60(\mathrm{~d}, J=8.3 \mathrm{~Hz}, 1 \mathrm{H}), 2.52$ (s, $3 \mathrm{H}) ;{ }^{13} \mathrm{C}$ NMR $\left(125 \mathrm{MHz} \mathrm{CDCl}_{3}\right) \delta 163.5,146.6,137.0,136.6,134.3,130.1,130.0$, 129.8, 129.7, 129.5, 129.0, 127.6, 83.1, 67.7, 60.1, 22.1; IR (thin film) 3066, 2923, 1766, 1596, 1476, 1375, 1310, 1231, 1191, 1173, 1092, 1014, 976, 910, 876, 815, 765, 736, 701, $662,569,547 \mathrm{~cm}^{-1}$; HRMS (FAB) calculated for $\mathrm{C}_{23} \mathrm{H}_{19} \mathrm{Cl}_{3} \mathrm{NO}_{3} \mathrm{~S}_{2}, 526.9872 \mathrm{~m} / \mathrm{z}(\mathrm{M}+$ H) ${ }^{+}$; observed 526.9897.

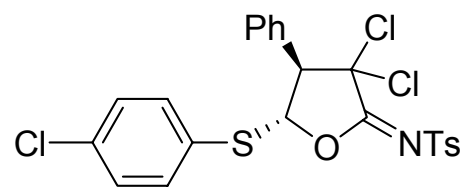

Lactone Imine $7 \mathrm{~g}$

N-[3,3-Dichloro-5-(4-chloro-phenylsulfanyl)-4-phenyl-dihydro-furan-2-ylidene]-4methyl-benzenesulfonamide (Table 1, entry g)

${ }^{1} \mathrm{H}$ NMR $\left(500 \mathrm{MHz} \mathrm{CDCl}_{3}\right) \delta 7.96(\mathrm{~d}, J=8.3 \mathrm{~Hz}, 2 \mathrm{H}), 7.51-7.40(\mathrm{~m}, 7 \mathrm{H}), 7.37(\mathrm{~d}, J=$ 8.1 Hz, 2 H), 7.31-7.29 (m, 2 H), 6.13 (d, $J=10.4$ Hz, 1 H), 3.67 (d, $J=10.4$ Hz, 1 H), $2.48(\mathrm{~s}, 3 \mathrm{H}) ;{ }^{13} \mathrm{C}$ NMR $\left(125 \mathrm{MHz} \mathrm{CDCl}_{3}\right) \delta 164.4,144.4,137.4,137.0,136.6,130.11$, $130.07,129.8,129.7,129.1,128.3,127.8,126.4,92.5,84.0,61.3,21.9$; IR (thin film) 2923, 1667, 1477, 1335, 1238, 1164, 1093, 1014, 910, 814, 741, $698 \mathrm{~cm}^{-1}$; HRMS (FAB) calculated for $\mathrm{C}_{23} \mathrm{H}_{19} \mathrm{Cl}_{3} \mathrm{NO}_{3} \mathrm{~S}_{2}, 525.9872 \mathrm{~m} / z(\mathrm{M}+\mathrm{H})^{+}$; observed 525.9879 . 


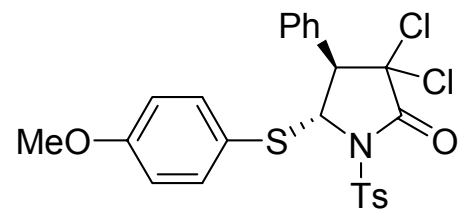

Lactam 6h

\section{3,3-Dichloro-5-(4-methoxy-phenylsulfanyl)-4-phenyl-1-(toluene-4-sulfonyl)- pyrrolidin-2-one (Table 1, entry $h$ )}

${ }^{1} \mathrm{H}$ NMR (500 MHz $\left.\mathrm{CDCl}_{3}\right) \delta 8.21(\mathrm{~d}, J=8.4 \mathrm{~Hz}, 2 \mathrm{H}), 7.45-7.43(\mathrm{~m}, 5 \mathrm{H}), 7.28-7.26(\mathrm{~m}$, $2 \mathrm{H}), 6.94(\mathrm{~d}, J=8.8 \mathrm{~Hz}, 2 \mathrm{H}), 6.76(\mathrm{~d}, J=8.8 \mathrm{~Hz}, 2 \mathrm{H}), 5.61(\mathrm{~d}, J=8.5 \mathrm{~Hz}, 1 \mathrm{H}), 3.80$ (s, $3 \mathrm{H}), 3.50(\mathrm{~d}, J=8.7 \mathrm{~Hz}, 1 \mathrm{H}), 2.50(\mathrm{~s}, 3 \mathrm{H}) ;{ }^{13} \mathrm{C} \mathrm{NMR}\left(125 \mathrm{MHz} \mathrm{CDCl}_{3}\right) \delta 163.7,161.3$, $146.4,138.1,134.5,130.2$, 129.9, 129.7, 129.5, 128.9, 118.5, 115.1, 83.5, 67.3, 58.8, 55.5, 22.0; IR (thin film) 2948, 1762, 1587, 1494, 1370, 1289, 1247, 1169, 1087, 1025, 973, $911,875,659 \mathrm{~cm}^{-1}$; HRMS (FAB) calculated for $\mathrm{C}_{24} \mathrm{H}_{22} \mathrm{Cl}_{2} \mathrm{NO}_{4} \mathrm{~S}_{2}, 522.0367 \mathrm{~m} / \mathrm{z}(\mathrm{M}+$ $\mathrm{H})^{+}$; observed 522.0388 .

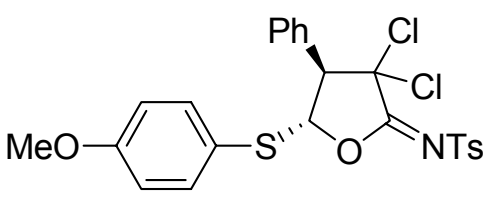

Lactone Imine $7 \mathrm{~h}$

N-[3,3-Dichloro-5-(4-methoxy-phenylsulfanyl)-4-phenyl-dihydro-furan-2-ylidene]-4methyl-benzenesulfonamide (Table 1 , entry $h$ )

${ }^{1} \mathrm{H}$ NMR $\left(500 \mathrm{MHz} \mathrm{CDCl}_{3}\right) \delta 7.98(\mathrm{~d}, J=8.3 \mathrm{~Hz}, 2 \mathrm{H}), 7.49-7.37$ (m, $\left.5 \mathrm{H}\right), 7.30-7.28(\mathrm{~m}$, $2 \mathrm{H}), 6.93(\mathrm{~d}, J=8.7 \mathrm{~Hz}, 2 \mathrm{H}), 6.08$ (d, $J=10.4 \mathrm{~Hz}, 1 \mathrm{H}), 3.88$ (s, $3 \mathrm{H}), 3.61$ (d, $J=10.4$ $\mathrm{Hz}, 1 \mathrm{H}), 2.48$ (s, $3 \mathrm{H}) ;{ }^{13} \mathrm{C}$ NMR (125 MHz $\left.\mathrm{CDCl}_{3}\right) \delta 164.8,161.6$, 144.3, 137.7, 137.4, 129.93, 129.90, 129.6, 129.0, 128.6, 127.9, 117.5, 115.3, 93.2, 84.3, 60.9, 55.6, 21.9; IR (thin film) 2947, 1664, 1588, 1490, 1331, 1244, 1157, 1086, 1024, $742 \mathrm{~cm}^{-1}$; HRMS (FAB) calculated for $\mathrm{C}_{24} \mathrm{H}_{22} \mathrm{Cl}_{2} \mathrm{NO}_{4} \mathrm{~S}_{2}, 522.0367 \mathrm{~m} / \mathrm{z}(\mathrm{M}+\mathrm{H})^{+}$; observed 522.0388. 


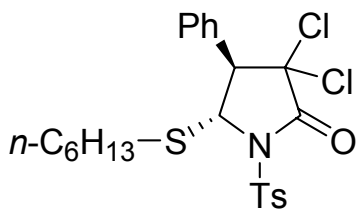

\section{Lactam 6i}

\section{3,3-Dichloro-5-hexylsulfanyl-4-phenyl-1-(toluene-4-sulfonyl)-pyrrolidin-2-one}

(Table 1, entry i)

${ }^{1} \mathrm{H}$ NMR $\left(500 \mathrm{MHz} \mathrm{CDCl}_{3}\right) \delta 8.13(\mathrm{~d}, J=8.3 \mathrm{~Hz}, 2 \mathrm{H}), 7.46-7.36(\mathrm{~m}, 7 \mathrm{H}), 5.46(\mathrm{~d}, J=$ $7.8 \mathrm{~Hz}, 1 \mathrm{H}), 3.85$ (d, $J=7.8 \mathrm{~Hz}, 1 \mathrm{H}), 2.80$ (t, $J=7.4 \mathrm{~Hz}, 2 \mathrm{H}), 2.48$ (s, $3 \mathrm{H}), 1.47-1.33$ $(\mathrm{m}, 2 \mathrm{H}), 1.25-1.12(\mathrm{~m}, 6 \mathrm{H}), 0.85(\mathrm{t}, J=7.0 \mathrm{~Hz}, 3 \mathrm{H}) ;{ }^{13} \mathrm{C} \mathrm{NMR}\left(125 \mathrm{MHz} \mathrm{CDCl}_{3}\right) \delta$ 163.8, 146.2 134.9, 131.1, 129.9, 129.8, 129.6, 129.2, 129.0, 82.9, 67.6, 62.8, 34.2, 31.4, 29.5, 28.4, 22.6, 22.0, 14.2; IR (thin film) 2925, 1763, 1372, 1225, 1171, 1083, 971, 697 , $658 \mathrm{~cm}^{-1}$; HRMS (FAB) calculated for $\mathrm{C}_{23} \mathrm{H}_{28} \mathrm{Cl}_{2} \mathrm{NO}_{3} \mathrm{~S}_{2}, 500.0888 \mathrm{~m} / z(\mathrm{M}+\mathrm{H})^{+}$; observed 500.0911 .

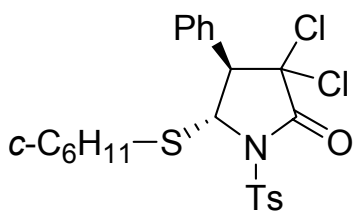

\section{Lactam 6j}

\section{3,3-Dichloro-5-cyclohexylsulfanyl-4-phenyl-1-(toluene-4-sulfonyl)-pyrrolidin-2-one}

\section{(Table 1, entry j)}

${ }^{1} \mathrm{H}$ NMR $\left(500 \mathrm{MHz} \mathrm{CDCl}_{3}\right) \delta 8.12(\mathrm{~d}, J=8.3 \mathrm{~Hz}, 2 \mathrm{H}), 7.44-7.42(\mathrm{~m}, 3 \mathrm{H}), 7.40(\mathrm{~d}, J=$ 8.5 Hz, $2 \mathrm{H}), 7.36-7.34$ (m, $2 \mathrm{H}), 5.50$ (d, J=7.0 Hz, $1 \mathrm{H}), 3.33-3.29$ (m, $1 \mathrm{H}), 2.48$ (s, 3 $\mathrm{H}), 1.99-1.96(\mathrm{~m}, 1 \mathrm{H}), 1.77-1.70(\mathrm{~m}, 2 \mathrm{H}), 1.60-1.54(\mathrm{~m}, 2 \mathrm{H}), 1.40-1.07(\mathrm{~m}, 5 \mathrm{H}) ;{ }^{13} \mathrm{C}$ NMR (125 MHz $\left.\mathrm{CDCl}_{3}\right) \delta 163.8,146.2,134.9,131.8,129.8,129.6,129.3,128.9,82.9$, 66.7, 63.9, 46.8, 34.9, 32.7, 26.1, 25.6, 25.5, 22.0; IR (thin film) 2926, 2896, 1760, 1595, 1446, 1371, 1166, 1067, 972, 740, 698, $658 \mathrm{~cm}^{-1}$; HRMS (FAB) calculated for $\mathrm{C}_{23} \mathrm{H}_{25} \mathrm{Cl}_{2} \mathrm{NO}_{3} \mathrm{~S}_{2}, 497.0653 \mathrm{~m} / z \mathrm{M}^{+}$; observed 497.0628 . 


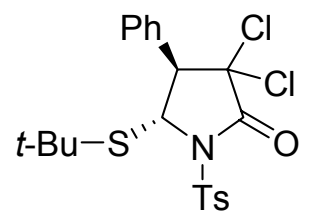

Lactam 6k

5-tert-Butylsulfanyl-3,3-dichloro-4-phenyl-1-(toluene-4-sulfonyl)-pyrrolidin-2-one

(Table 1, entry $k)$

${ }^{1} \mathrm{H}$ NMR $\left(500 \mathrm{MHz}_{\mathrm{CDCl}}\right) \delta 8.13(\mathrm{~d}, J=8.4 \mathrm{~Hz}, 2 \mathrm{H}), 7.41-7.39(\mathrm{~m}, 3 \mathrm{H})$, 7.23-7.21 (m, $2 \mathrm{H}), 5.48(\mathrm{~d}, J=2.3 \mathrm{~Hz}, 1 \mathrm{H}), 4.12(\mathrm{~d}, J=2.2 \mathrm{~Hz}, 1 \mathrm{H}), 2.49(\mathrm{~s}, 3 \mathrm{H}), 1.35(\mathrm{~s}, 9 \mathrm{H}) ;{ }^{13} \mathrm{C}$ NMR $\left(125 \mathrm{MHz} \mathrm{CDCl}_{3}\right) \delta 170.9,146.4,136.2,134.4,129.9,129.7,129.52,129.47$, 128.5, 66.8, 65.4, 46.2, 31.6, 30.0, 22.1; IR (thin film) 2963, 1763, 1595, 1496, 1458, 1370, $1328 \mathrm{~cm}^{-1}$; HRMS (FAB) calculated for $\mathrm{C}_{21} \mathrm{H}_{23} \mathrm{Cl}_{2} \mathrm{NO}_{3} \mathrm{~S}_{2}, 472.0575 \mathrm{~m} / \mathrm{z} \mathrm{M}^{+}$; observed 472.0597.<smiles>O=C1N([123I])[C@H](SBr)[C@@H](c2ccccc2)C1(Cl)Cl</smiles>

\section{Lactam 61}

\section{5-Benzylsulfanyl-3,3-dichloro-4-phenyl-1-(toluene-4-sulfonyl)-pyrrolidin-2-one}

(Table 1, entry 1 )

${ }^{1} \mathrm{H}$ NMR $\left(500 \mathrm{MHz} \mathrm{CDCl}_{3}\right) \delta 8.14(\mathrm{~d}, J=8.3 \mathrm{~Hz}, 2 \mathrm{H}), 7.42-7.17(\mathrm{~m}, 10 \mathrm{H}), 6.82(\mathrm{~d}, J=$ $7.4 \mathrm{~Hz}, 2 \mathrm{H}), 5.32$ (d, $J=8.2 \mathrm{~Hz}, 1 \mathrm{H}), 4.34(\mathrm{~d}, J=13.2 \mathrm{~Hz}, 1 \mathrm{H}), 3.88$ (d, $J=13.2 \mathrm{~Hz}, 1$ H), $3.69(\mathrm{~d}, J=8.2 \mathrm{~Hz}, 1 \mathrm{H}), 2.48(\mathrm{~s}, 3 \mathrm{H}) ;{ }^{13} \mathrm{C} \mathrm{NMR}\left(125 \mathrm{MHz} \mathrm{CDCl}_{3}\right) \delta 163.9,146.3$, $136.7,134.7,130.1,129.9,129.7,129.5,129.4,129.2,129.0,128.7,127.7,82.8,65.4$, 62.3, 38.1, 22.0; IR (thin film) 3032, 1764, 1597, 1495, 1372, 1229, 1174, 1087, 1074, 977, 764, 701, $662 \mathrm{~cm}^{-1}$; HRMS (FAB) calculated for $\mathrm{C}_{24} \mathrm{H}_{22} \mathrm{Cl}_{2} \mathrm{NO}_{3} \mathrm{~S}_{2}, 506.0418 \mathrm{~m} / z$ (M $+\mathrm{H})^{+}$; observed 506.0395. 


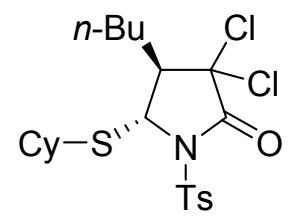

Lactam 9

4-Butyl-3,3-dichloro-5-cyclohexylsulfanyl-1-(toluene-4-sulfonyl)-pyrrolidin-2-one (Table 2, entry 1)

${ }^{1} \mathrm{H}$ NMR (500 MHz $\left.\mathrm{CDCl}_{3}\right) \delta 8.05(\mathrm{~d}, J=8.4 \mathrm{~Hz}, 2 \mathrm{H}), 7.36(\mathrm{~d}, J=8.1 \mathrm{~Hz}, 2 \mathrm{H}), 4.82(\mathrm{~d}$, $J=7.9 \mathrm{~Hz}, 1 \mathrm{H}), 3.40(\mathrm{~m}, 1 \mathrm{H}), 2.52(\mathrm{dt}, J=8.2,4.7 \mathrm{~Hz}, 1 \mathrm{H}), 2.46(\mathrm{~s}, 3 \mathrm{H}), 2.05-1.26$ (m, $16 \mathrm{H}) ;{ }^{13} \mathrm{C} \mathrm{NMR}\left(125 \mathrm{MHz} \mathrm{CDCl}_{3}\right) \delta 164.3,146.0,135.0,129.7,129.1,83.6,67.3,56.2$, $46.6,35.5,32.8,29.0,27.4,26.3,25.8,25.7,22.7,22.0,13.9$; IR (thin film) 2927, 2845, $1762,1593,1444,1372,1229,1173,1080,978,812,704,679,661,578 \mathrm{~cm}^{-1}$; HRMS (FAB) calculated for $\mathrm{C}_{21} \mathrm{H}_{30} \mathrm{Cl}_{2} \mathrm{NO}_{3} \mathrm{~S}_{2}, 478.1044 \mathrm{~m} / z(\mathrm{M}+\mathrm{H})^{+}$; observed 478.1034 .

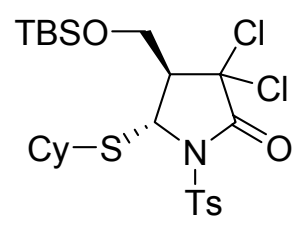

\section{Lactam 11}

4-(tert-Butyl-dimethyl-silanyloxymethyl)-3,3-dichloro-5-cyclohexylsulfanyl-1(toluene-4-sulfonyl)-pyrrolidin-2-one (Table 2, entry 2)

${ }^{1} \mathrm{H}$ NMR $\left(500 \mathrm{MHz}_{\mathrm{CDCl}}\right) \delta 8.06(\mathrm{~d}, J=8.4 \mathrm{~Hz}, 2 \mathrm{H}), 7.36(\mathrm{~d}, J=8.2 \mathrm{~Hz}, 2 \mathrm{H}), 5.45(\mathrm{~d}$, $J=2.3 \mathrm{~Hz}, 1 \mathrm{H}), 4.02(\mathrm{dd}, J=10.6,5.1 \mathrm{~Hz}, 1 \mathrm{H}), 3.70(\mathrm{dd}, J=10.6,7.1 \mathrm{~Hz}, 1 \mathrm{H}), 3.33-$ $3.28(\mathrm{~m}, 1 \mathrm{H}), 3.08-3.05(\mathrm{~m}, 1 \mathrm{H}), 2.46(\mathrm{~s}, 3 \mathrm{H}), 2.12-1.26(\mathrm{~m}, 10 \mathrm{H}), 0.94(\mathrm{~s}, 9 \mathrm{H}), 0.13$ $(\mathrm{s}, 6 \mathrm{H}) ;{ }^{13} \mathrm{C} \mathrm{NMR}\left(125 \mathrm{MHz} \mathrm{CDCl}_{3}\right) \delta 163.6,146.1,134.6,129.8,129.2,81.7,64.0$, $61.4,60.1,47.2,35.5,32.8,26.2,26.0,25.7,22.0,18.5,-5.4$; IR (thin film) 2930, 2856, 1764, 1597, 1471, 1449, 1376, 1333, 1258, 1190, 1176, 1115, 1084, 997, 972, 837, 813, 780, 703, 679, $662 \mathrm{~cm}^{-1}$; HRMS (FAB) calculated for $\mathrm{C}_{24} \mathrm{H}_{38} \mathrm{Cl}_{2} \mathrm{NO}_{4} \mathrm{~S}_{2} \mathrm{Si}, 566.1389 \mathrm{~m} / \mathrm{z}$ $(\mathrm{M}+\mathrm{H})^{+}$; observed 566.1367 . 


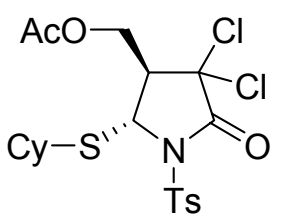

Lactam 13

Acetic acid 4,4-dichloro-2-cyclohexylsulfanyl-5-oxo-1-(toluene-4-sulfonyl)pyrrolidin-3-ylmethyl ester (Table 2, entry 3)

${ }^{1} \mathrm{H}$ NMR (500 MHz $\left.\mathrm{CDCl}_{3}\right) \delta 8.05(\mathrm{~d}, J=8.4 \mathrm{~Hz}, 2 \mathrm{H}), 7.36(\mathrm{~d}, J=8.2 \mathrm{~Hz}, 2 \mathrm{H}), 5.21$ (d, $J=4.8 \mathrm{~Hz}, 1 \mathrm{H}), 4.52(\mathrm{dd}, J=11.9,5.8 \mathrm{~Hz}, 1 \mathrm{H}), 4.45(\mathrm{dd}, J=11.8,4.7 \mathrm{~Hz}, 1 \mathrm{H}), 3.38$ 3.33 (m, $1 \mathrm{H}), 3.13$ (dd, $J=10.3,4.8 \mathrm{~Hz}, 1 \mathrm{H}), 2.45$ (s, $3 \mathrm{H}), 2.09$ (s, $3 \mathrm{H}), 2.04-1.24$ (m, $10 \mathrm{H}) ;{ }^{13} \mathrm{C} \mathrm{NMR}\left(125 \mathrm{MHz} \mathrm{CDCl}_{3}\right) \delta 170.6,163.4,146.4,134.6,130.0,129.3,81.4,63.9$, $61.5,56.8,47.1,35.6,32.8,26.3,25.9,25.8,22.1,20.9$; IR (thin film) 2930, 2853, 1762, 1596, 1449, 1373, 1226, 1175, 1085, 813, 664, $577 \mathrm{~cm}^{-1}$; HRMS (FAB) calculated for $\mathrm{C}_{20} \mathrm{H}_{26} \mathrm{Cl}_{2} \mathrm{NO}_{5} \mathrm{~S}_{2}, 492.0473 \mathrm{~m} / \mathrm{z}(\mathrm{M}-\mathrm{H})^{+}$; observed 492.0480 .

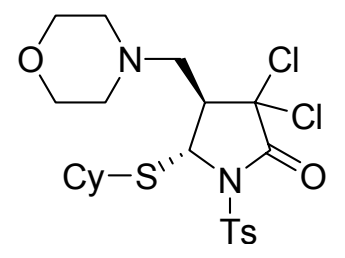

Lactam 15

\section{3,3-Dichloro-5-cyclohexylsulfanyl-4-morpholin-4-ylmethyl-1-(toluene-4-sulfonyl)-} pyrrolidin-2-one (Table 2, entry 4)

${ }^{1} \mathrm{H}$ NMR $\left(500 \mathrm{MHz}_{\mathrm{CDCl}}\right) \delta 8.05(\mathrm{~d}, J=8.3 \mathrm{~Hz}, 2 \mathrm{H}), 7.37(\mathrm{~d}, J=8.3 \mathrm{~Hz}, 2 \mathrm{H}), 5.55(\mathrm{~d}$, $J=2.8 \mathrm{~Hz}, 1 \mathrm{H}), 3.76-3.69$ (m, $4 \mathrm{H}), 3.43-3.39$ (m, $1 \mathrm{H}), 3.11-3.07$ (m, $1 \mathrm{H}), 2.77$ (dd, $J$ $=13.0,5.7 \mathrm{~Hz}, 1 \mathrm{H}), 2.60-2.59(\mathrm{~m}, 2 \mathrm{H}), 2.49-2.45(\mathrm{~m}, 4 \mathrm{H}), 2.36(\mathrm{dd}, J=12.8,2.5 \mathrm{~Hz}, 1$ $\mathrm{H}), 2.19-1.26(\mathrm{~m}, 11 \mathrm{H}) ;{ }^{13} \mathrm{C} \mathrm{NMR}\left(125 \mathrm{MHz} \mathrm{CDCl}_{3}\right) \delta 163.6,146.2,134.4,129.8,129.2$, $82.6,67.0,64.5,58.4,55.6,53.9,46.9,35.2,32.7,26.3,25.8,25.7,22.0$; IR (thin film) 2929, 2853, 1761, 1596, 1449, 1374, 1175, 1118, 1090, 972, 872, 813, 736, 681, 662, 578, $546 \mathrm{~cm}^{-1}$; HRMS (FAB) calculated for $\mathrm{C}_{22} \mathrm{H}_{31} \mathrm{Cl}_{2} \mathrm{~N}_{2} \mathrm{O}_{4} \mathrm{~S}_{2}, 521.1102 \mathrm{~m} / \mathrm{z}(\mathrm{M}+\mathrm{H})^{+}$; observed 521.1044 . 


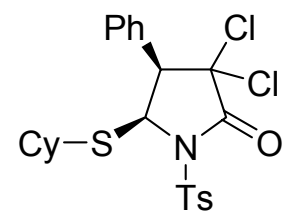

Lactam 17

\section{3,3-Dichloro-5-cyclohexylsulfanyl-4-phenyl-1-(toluene-4-sulfonyl)-pyrrolidin-2-one}

(Table 2, entry 5)

${ }^{1} \mathrm{H}$ NMR $\left(500 \mathrm{MHz} \mathrm{CDCl}_{3}\right) \delta 8.13(\mathrm{~d}, J=8.4 \mathrm{~Hz}, 2 \mathrm{H}), 7.64(\mathrm{~d}, J=6.8 \mathrm{~Hz}, 2 \mathrm{H}), 7.44-$ $7.38(\mathrm{~m}, 5 \mathrm{H}), 5.61(\mathrm{~d}, J=6.4 \mathrm{~Hz}, 1 \mathrm{H}), 4.26(\mathrm{~d}, J=6.4 \mathrm{~Hz}, 1 \mathrm{H}), 2.91-2.87(\mathrm{~m}, 1 \mathrm{H})$, 2.49 (s, $3 \mathrm{H}), 2.01-1.98$ (m, $1 \mathrm{H}), 1.71-1.68$ (m, $1 \mathrm{H}), 1.57-0.88(\mathrm{~m}, 8 \mathrm{H}) ;{ }^{13} \mathrm{C} \mathrm{NMR}(125$ $\left.\mathrm{MHz} \mathrm{CDCl}_{3}\right) \delta 161.0,148.7,140.9,136.3,129.5,128.2,126.3,125.7,125.4,97.1,46.2$, 36.0, 33.8, 33.7, 26.7, 23.8, 20.9; IR (thin film) 2916, 1764, 1598, 1372, 1173, 1002, 968, 872, 813, 694, 662, $544 \mathrm{~cm}^{-1}$; HRMS (FAB) calculated for $\mathrm{C}_{23} \mathrm{H}_{26} \mathrm{Cl}_{2} \mathrm{NO}_{3} \mathrm{~S}_{2}, 498.0731$ $m / z(\mathrm{M}+\mathrm{H})^{+}$; observed 498.0745.

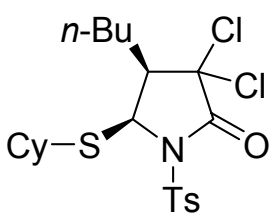

Lactam 19

\section{4-Butyl-3,3-dichloro-5-cyclohexylsulfanyl-1-(toluene-4-sulfonyl)-pyrrolidin-2-one} (Table 2, entry 6)

${ }^{1} \mathrm{H}$ NMR $\left(500 \mathrm{MHz}_{\mathrm{CDCl}}\right) \delta 8.06(\mathrm{~d}, J=8.4 \mathrm{~Hz}, 2 \mathrm{H}), 7.36(\mathrm{~d}, J=8.1 \mathrm{~Hz}, 2 \mathrm{H}), 5.47(\mathrm{~d}$, $J=7.0 \mathrm{~Hz}, 1 \mathrm{H}), 3.32-3.28$ (m, $1 \mathrm{H}), 2.99-2.95$ (m, $1 \mathrm{H}), 2.46$ (s, $3 \mathrm{H}), 2.05-1.27$ (m, 16 $\mathrm{H}) ;{ }^{13} \mathrm{C} \mathrm{NMR}\left(125 \mathrm{MHz} \mathrm{CDCl}_{3}\right) \delta 164.2,146.3,134.6,129.9,129.3,84.1,66.8,54.8$, $46.9,35.3,33.3,28.7,26.5,26.3,25.8,23.0,22.1,14.1$; IR (thin film) 2930, 2855, 1760, 1597, 1449, 1372, 1322, 1190, 1174, 1088, $972 \mathrm{~cm}^{-1}$; HRMS (FAB) calculated for $\mathrm{C}_{21} \mathrm{H}_{30} \mathrm{Cl}_{2} \mathrm{NO}_{3} \mathrm{~S}_{2}, 478.1044 \mathrm{~m} / z(\mathrm{M}+\mathrm{H})^{+}$; observed 478.1032 . 


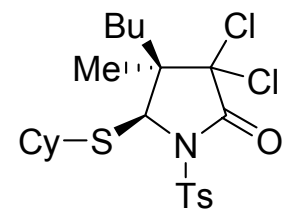

Lactam 21

\section{4-Butyl-3,3-dichloro-5-cyclohexylsulfanyl-4-methyl-1-(toluene-4-sulfonyl)-}

\section{pyrrolidin-2-one (Table 2, entry 7)}

${ }^{1} \mathrm{H}$ NMR (500 MHz CDCl 3$) \delta 8.04(\mathrm{~d}, J=8.4 \mathrm{~Hz}, 2 \mathrm{H}), 7.36(\mathrm{~d}, J=8.2 \mathrm{~Hz}, 2 \mathrm{H}), 5.10(\mathrm{~s}$, $1 \mathrm{H}), 3.30-3.26(\mathrm{~m}, 1 \mathrm{H}), 2.46(\mathrm{~s}, 3 \mathrm{H}), 2.10-2.02(\mathrm{~m}, 3 \mathrm{H}), 1.82-1.26(\mathrm{~m}, 16 \mathrm{H}), 0.92(\mathrm{t}, J$

$=7.1 \mathrm{~Hz}, 3 \mathrm{H}) ;{ }^{13} \mathrm{C}$ NMR $\left(125 \mathrm{MHz} \mathrm{CDCl}_{3}\right) \delta$ 164.0, 146.1, 134.6, 129.8, 129.1, 89.1, $73.8,52.5,47.2,35.4,34.0,33.1,26.3,25.8,25.75,25.67,23.8,23.5,22.0,14.1$; IR (thin film) $2931,2855,1760,1597,1450,1372,1325,1240,1174,1089,1000,911,835,814$, 733, $661 \mathrm{~cm}^{-1}$; HRMS (FAB) calculated for $\mathrm{C}_{22} \mathrm{H}_{32} \mathrm{Cl}_{2} \mathrm{NO}_{3} \mathrm{~S}_{2}, 492.1201 \mathrm{~m} / z(\mathrm{M}+\mathrm{H})^{+}$; observed 492.1187.

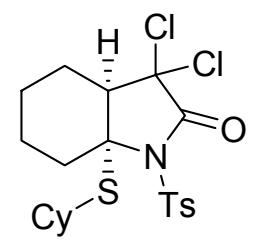

Lactam 23

\section{3,3-Dichloro-7a-cyclohexylsulfanyl-1-(toluene-4-sulfonyl)-octahydro-indol-2-one} (Table 2, entry 8)

${ }^{1} \mathrm{H}$ NMR $\left(500 \mathrm{MHz} \mathrm{CDCl}_{3}\right) \delta 8.00(\mathrm{~d}, J=8.4 \mathrm{~Hz}, 2 \mathrm{H}), 7.35(\mathrm{~d}, J=8.1 \mathrm{~Hz}, 2 \mathrm{H}), 2.92-$ $2.88(\mathrm{~m}, 2 \mathrm{H}), 2.74-2.69(\mathrm{~m}, 1 \mathrm{H}), 2.45$ (s, $3 \mathrm{H}), 2.28-1.17(\mathrm{~m}, 17 \mathrm{H}) ;{ }^{13} \mathrm{C}$ NMR $(125$ $\left.\mathrm{MHz} \mathrm{CDCl}_{3}\right) \delta 164.2,145.9,134.8,129.6,129.5,83.6,77.5,51.4,43.3,35.1,34.8,34.0$, 26.4, 26.3, 25.5, 21.95, 21.86, 20.8, 20.6; IR (thin film) 2930, 2854, 1754, 1597, 1449, 1375, 1250, 1177, 1084, 995, 966, 908, 874, 733, 663, $596 \mathrm{~cm}^{-1}$; HRMS (FAB) calculated for $\mathrm{C}_{21} \mathrm{H}_{28} \mathrm{Cl}_{2} \mathrm{NO}_{3} \mathrm{~S}_{2}, 476.0888 \mathrm{~m} / z(\mathrm{M}+\mathrm{H})^{+}$; observed 476.0860. 


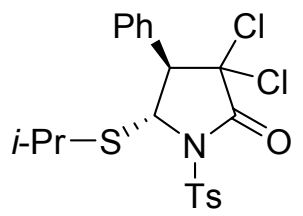

Lactam 24

\section{3,3-Dichloro-5-isopropylsulfanyl-4-phenyl-1-(toluene-4-sulfonyl)-pyrrolidin-2-one} (Scheme 2)

${ }^{1} \mathrm{H}$ NMR $\left(400 \mathrm{MHz} \mathrm{CDCl}_{3}\right) \delta 8.10(\mathrm{~d}, J=8.4 \mathrm{~Hz}, 2 \mathrm{H}), 7.45-7.33(\mathrm{~m}, 7 \mathrm{H}), 5.47(\mathrm{~d}, J=$ $7.1 \mathrm{~Hz}, 1 \mathrm{H}), 3.83$ (d, $J=7.1 \mathrm{~Hz}, 1 \mathrm{H}), 3.56$ (sept, $J=6.7 \mathrm{~Hz}, 1 \mathrm{H}), 2.47$ (s, $3 \mathrm{H}), 1.23$ (d, $J=6.9 \mathrm{~Hz}, 3 \mathrm{H}), 1.11(\mathrm{~d}, J=6.9 \mathrm{~Hz}, 3 \mathrm{H}) ;{ }^{13} \mathrm{C} \mathrm{NMR}\left(100 \mathrm{MHz} \mathrm{CDCl}_{3}\right) \delta 163.8,146.2$, 134.8, 131.7, 129.84, 129.78, 129.6, 129.3, 128.9, 82.9, 67.1, 63.6, 38.5, 24.9, 22.7, 22.0; IR (thin film) 2925, 2890, 1762, 1590, 1440, 1370, 1166, 1060, 975, 740, $695 \mathrm{~cm}^{-1}$; LRMS (FAB) calculated for $\mathrm{C}_{20} \mathrm{H}_{21} \mathrm{Cl}_{2} \mathrm{NO}_{3} \mathrm{~S}_{2}, 458 \mathrm{~m} / z(\mathrm{M}+\mathrm{H})^{+}$; observed 458, 382, 155 , 136; HRMS (FAB) calculated for $\mathrm{C}_{20} \mathrm{H}_{22} \mathrm{Cl}_{2} \mathrm{NO}_{3} \mathrm{~S}_{2}, 458.0418 \mathrm{~m} / z(\mathrm{M}+\mathrm{H})^{+}$; observed 458.0433.

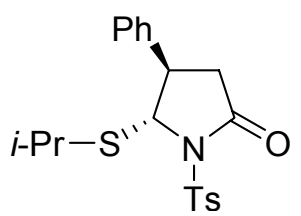

\section{Lactam 25}

\section{5-Isopropylsulfanyl-4-phenyl-1-(toluene-4-sulfonyl)-pyrrolidin-2-one (Scheme 2)}

${ }^{1} \mathrm{H}$ NMR (400 MHz $\mathrm{CDCl}_{3}$ ) $\delta$ 7.92-7.89 (m, $\left.2 \mathrm{H}\right), 7.30-7.28(\mathrm{~m}, 5 \mathrm{H}), 7.10-7.06(\mathrm{~m}, 2 \mathrm{H})$, $5.31(\mathrm{~d}, J=1.0 \mathrm{~Hz}, 1 \mathrm{H}), 3.56$ (d, $J=8.2 \mathrm{~Hz}, 1 \mathrm{H}), 3.40$ (sept, $J=6.7 \mathrm{~Hz}, 1 \mathrm{H}), 3.17$ (dd, $J=17.7,8.3 \mathrm{~Hz}, 1 \mathrm{H}), 2.55(\mathrm{dd}, J=17.7,1.0 \mathrm{~Hz}, 1 \mathrm{H}), 2.45(\mathrm{~s}, 3 \mathrm{H}), 1.35(\mathrm{dd}, J=7.2$, $6.9 \mathrm{~Hz}, 6 \mathrm{H}) ;{ }^{13} \mathrm{C}$ NMR $\left(100 \mathrm{MHz} \mathrm{CDCl}_{3}\right) \delta 172.3,145.4,141.3,135.2,129.5,129.2$, 128.0, 126.4, 71.2, 48.1, 37.8, 36.9, 24.6, 23.4, 21.9; IR (thin film) 2935, 2853, 1745, 1595, 1492, 1455, 1360, 1175, 1090, 960, 941, 910, 813, 735, $700 \mathrm{~cm}^{-1}$; HRMS (FAB) calculated for $\mathrm{C}_{20} \mathrm{H}_{24} \mathrm{NO}_{3} \mathrm{~S}_{2}, 390.1198 \mathrm{~m} / z(\mathrm{M}+\mathrm{H})^{+}$; observed 390.1172 . 


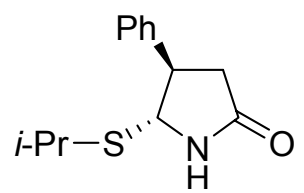

Lactam 26

\section{5-Isopropylsulfanyl-4-phenyl-pyrrolidin-2-one (Scheme 2)}

${ }^{1} \mathrm{H}$ NMR $\left(400 \mathrm{MHz} \mathrm{CDCl}_{3}\right) \delta$ 7.31-7.18 (m, $\left.5 \mathrm{H}\right), 6.82($ broad s, $1 \mathrm{H}), 4.65(\mathrm{~d}, J=5.1 \mathrm{~Hz}$, $1 \mathrm{H}), 3.47-3.39$ (m, $1 \mathrm{H}), 2.95$ (sept, $J=6.7 \mathrm{~Hz}, 1 \mathrm{H}), 2.84(\mathrm{dd}, J=17.3,9.2 \mathrm{~Hz}, 1 \mathrm{H})$, $2.47(\mathrm{dd}, J=17.7,6.7 \mathrm{~Hz}, 1 \mathrm{H}), 1.19(\mathrm{~d}, J=6.7 \mathrm{~Hz}, 3 \mathrm{H}), 1.15(\mathrm{~d}, J=6.7 \mathrm{~Hz}, 3 \mathrm{H}) ;{ }^{13} \mathrm{C}$ NMR $\left(100 \mathrm{MHz} \mathrm{CDCl}_{3}\right) \delta 176.5,141.4,129.2,127.7,127.1,65.9,48.5,38.0,35.6,24.1$, 24.1; IR (thin film) 3300, 2926, 2896, 1760, 1595, 1446, 1371, 1166, 1067, 972, 740, 698, $658 \mathrm{~cm}^{-1}$; LRMS (ES) calculated for $\mathrm{C}_{13} \mathrm{H}_{17} \mathrm{NOS}, 236 \mathrm{~m} / z(\mathrm{M}+\mathrm{H})^{+}$; observed 236, 177 , 160; HRMS (FAB) calculated for $\mathrm{C}_{13} \mathrm{H}_{18} \mathrm{NOS}, 236.1109 \mathrm{~m} / z(\mathrm{M}+\mathrm{H})^{+}$; observed 236.1122. General Procedure for the Synthesis of Sulfilimines (synthesis of sulfilimine 5a of Table 1 as an example)

To a dry flask equipped with magnetic stir bar was added phenyl styryl sulfide $(1.0 \mathrm{~g}$, $4.71 \mathrm{mmol}), \mathrm{MeCN} 25 \mathrm{~mL}$ and chloramine-T trihydrate $(1.7 \mathrm{~g}, 6.12 \mathrm{mmol})$. Stir at room temperature over 24 hours and sample the reaction for TLC monitoring. Upon disappearance of the sulfide, the reaction mixture was diluted with $\mathrm{CHCl}_{3}$ and filtered through a celite plug. The filtrate was concentrated in vacuo to give a crude product, which was purified via silica gel chromatography with ethyl acetate / hexanes $(75: 25)$ to give sulfilimine $5 \mathbf{a}(1.65 \mathrm{~g}, 92 \%)$.

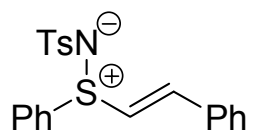

\section{Sulfilimine 5a (Table 1, entry a)}

${ }^{1} \mathrm{H}$ NMR $\left(500 \mathrm{MHz} \mathrm{CDCl}_{3}\right) \delta 7.79(\mathrm{~d}, J=8.2 \mathrm{~Hz}, 2 \mathrm{H}), 7.71-7.70(\mathrm{~m}, 2 \mathrm{H})$, 7.55-7.48 (m, $3 \mathrm{H}), 7.39-7.36(\mathrm{~m}, 6 \mathrm{H}), 7.17(\mathrm{~d}, J=8.1 \mathrm{~Hz}, 2 \mathrm{H}), 6.60$ (d, $J=15.2 \mathrm{~Hz}, 1 \mathrm{H}), 2.32$ (s, 3 $\mathrm{H}) ;{ }^{13} \mathrm{C}$ NMR $\left(125 \mathrm{MHz} \mathrm{CDCl}_{3}\right) \delta 145.0,141.9,141.7,135.7,133.0,132.6,131.2,130.2$, 129.5, 129.3, 128.4, 127.1, 126.6, 122.9; IR (thin film) 1597, 1496, 1279, 1248, 1134, $1090,979,935,807 \mathrm{~cm}^{-1}$; HRMS (FAB) calculated for $\mathrm{C}_{21} \mathrm{H}_{19} \mathrm{NO}_{2} \mathrm{~S}_{2}, 382.0935 \mathrm{~m} / \mathrm{z}(\mathrm{M}+$ $\mathrm{H})^{+}$; observed 382.0919. 


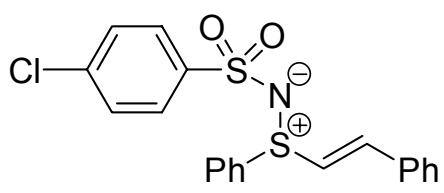

\section{Sulfilimine 5b (Table 1, entry b)}

${ }^{1} \mathrm{H}$ NMR (500 MHz CDCl $) \delta 7.81(\mathrm{~d}, J=8.6 \mathrm{~Hz}, 2 \mathrm{H})$, 7.69-7.66 (m, $\left.2 \mathrm{H}\right)$, 7.54-7.38 (m, $11 \mathrm{H}), 6.64(\mathrm{~d}, J=15.2 \mathrm{~Hz}, 1 \mathrm{H}) ;{ }^{13} \mathrm{C}$ NMR $\left(125 \mathrm{MHz} \mathrm{CDCl}_{3}\right) \delta 142.9,141.9,137.4$, 135.0, 132.54, 132.48, 131.0, 130.1, 129.1, 128.8, 128.2, 127.8, 126.8, 122.1; IR (thin film) 3060, 1593, 1476, 1446, 1301, 1274, 1145, 1089, 968, 744, 686, 620, $569 \mathrm{~cm}^{-1}$; HRMS (FAB) calculated for $\mathrm{C}_{20} \mathrm{H}_{16} \mathrm{ClN}_{2} \mathrm{O}_{2} \mathrm{~S}_{2}, 402.0389 \mathrm{~m} / \mathrm{z}(\mathrm{M}+\mathrm{H})^{+}$; observed 402.0367 .

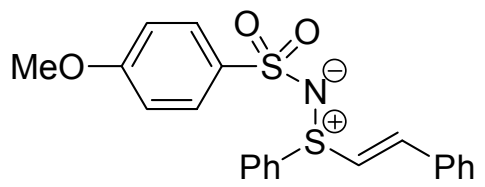

\section{Sulfilimine 5c (Table 1, entry c)}

${ }^{1} \mathrm{H}$ NMR $\left(500 \mathrm{MHz} \mathrm{CDCl}_{3}\right) \delta 7.83(\mathrm{~d}, J=8.8 \mathrm{~Hz}, 2 \mathrm{H}), 7.69(\mathrm{~d}, J=8.2 \mathrm{~Hz}, 2 \mathrm{H}), 7.50$ $7.33(\mathrm{~m}, 9 \mathrm{H}), 6.83(\mathrm{~d}, J=8.9 \mathrm{~Hz}, 2 \mathrm{H}), 6.61(\mathrm{~d}, J=15.2 \mathrm{~Hz}, 1 \mathrm{H}) ;{ }^{13} \mathrm{C} \mathrm{NMR}(125 \mathrm{MHz}$ $\left.\mathrm{CDCl}_{3}\right) \delta 161.8,141.7,136.4,135.5,132.8,132.4,130.9,130.1,129.13,129.12,138.4$, 128.2, 126.9, 122.6, 113.8; IR (thin film) 3060, 2927, 1597, 1497, 1446, 1310, 1286, 1257, 1140, 1089, 964, 833, 806, 742, 687, $577 \mathrm{~cm}^{-1}$; HRMS (FAB) calculated for $\mathrm{C}_{21} \mathrm{H}_{19} \mathrm{NO}_{3} \mathrm{~S}_{2}, 398.0885 \mathrm{~m} / \mathrm{z}(\mathrm{M}+\mathrm{H})^{+}$; observed 398.0906.

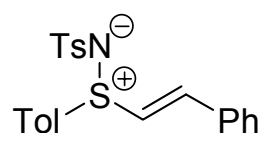

\section{Sulfilimine $5 f$ (Table 1, entry f)}

${ }^{1} \mathrm{H}$ NMR (500 MHz CDCl 3 ) $\delta 7.78(\mathrm{~d}, J=8.2 \mathrm{~Hz}, 2 \mathrm{H}), 7.56(\mathrm{~d}, J=8.3 \mathrm{~Hz}, 2 \mathrm{H}), 7.35(\mathrm{~m}$, $5 \mathrm{H}), 7.34(\mathrm{~d}, J=15.1 \mathrm{~Hz}, 1 \mathrm{H}), 7.27(\mathrm{~d}, J=8.4 \mathrm{~Hz}, 2 \mathrm{H}), 7.15(\mathrm{~d}, J=8.2 \mathrm{~Hz}, 2 \mathrm{H}), 6.58$ $(\mathrm{d}, J=15.1 \mathrm{~Hz}, 1 \mathrm{H}), 2.37(\mathrm{~s}, 3 \mathrm{H}), 2.30(\mathrm{~s}, 3 \mathrm{H}) ;{ }^{13} \mathrm{C} \mathrm{NMR}\left(125 \mathrm{MHz} \mathrm{CDCl}_{3}\right) \delta$ 143.4, 141.7, 141.6, 141.1, 132.8, 132.1, 130.8, 130.7, 129.3, 129.1, 128.2, 127.0, 126.4, 122.8, 21.5, 21.4; IR (thin film) 3033, 2923, 2251, 1597, 1493, 1448, 1400, 1296, 1281, 1142, 1089, 1020, 970, 911, 812, 735, 657, 571, $550 \mathrm{~cm}^{-1}$; HRMS (FAB) calculated for $\mathrm{C}_{22} \mathrm{H}_{21} \mathrm{NO}_{2} \mathrm{~S}_{2}, 396.1092 \mathrm{~m} / \mathrm{z}(\mathrm{M}+\mathrm{H})^{+}$; observed 396.1117 . 


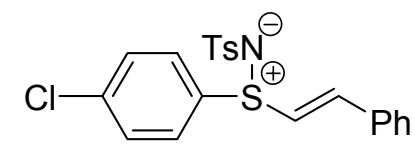

\section{Sulfilimine 5g (Table 1, entry g)}

${ }^{1} \mathrm{H}$ NMR (500 MHz CDCl $) \delta 7.76(\mathrm{~d}, J=8.2 \mathrm{~Hz}, 2 \mathrm{H}), 7.63(\mathrm{~d}, J=8.7 \mathrm{~Hz}, 2 \mathrm{H}), 7.43(\mathrm{~d}$, $J=8.7 \mathrm{~Hz}, 2 \mathrm{H}), 7.38-7.35(\mathrm{~m}, 6 \mathrm{H}), 7.16(\mathrm{~d}, J=8.1 \mathrm{~Hz}, 2 \mathrm{H}), 6.61(\mathrm{~d}, J=15.2 \mathrm{~Hz}, 1 \mathrm{H})$, $2.31(\mathrm{~s}, 3 \mathrm{H}) ;{ }^{13} \mathrm{C}$ NMR $\left(125 \mathrm{MHz} \mathrm{CDCl}_{3}\right) \delta 142.2,142.0,141.4,138.9,134.0,132.6$, 131.2, 130.3, 129.4, 129.2, 128.3, 128.2, 126.4, 122.2, 21.5; IR (thin film) 3018, 2923, 1600, 1573, 1475, 1449, 1297, 1282, 1141, 1088, 966, 815, 747, 657, 578, $552 \mathrm{~cm}^{-1}$; HRMS (FAB) calculated for $\mathrm{C}_{22} \mathrm{H}_{18} \mathrm{ClNO}_{2} \mathrm{~S}_{2}, 416.0546 \mathrm{~m} / z(\mathrm{M}+\mathrm{H})^{+}$; observed 416.0567.

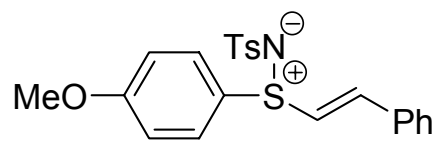

\section{Sulfilimine 5h (Table 1, entry h)}

${ }^{1} \mathrm{H}$ NMR (500 MHz CDCl $) \delta 7.77(\mathrm{~d}, J=8.2 \mathrm{~Hz}, 2 \mathrm{H}), 7.61(\mathrm{~d}, J=8.9 \mathrm{~Hz}, 2 \mathrm{H}), 7.37(\mathrm{~m}$, $5 \mathrm{H}), 7.34(\mathrm{~d}, J=15.2 \mathrm{~Hz}, 1 \mathrm{H}), 7.15(\mathrm{~d}, J=8.1 \mathrm{~Hz}, 2 \mathrm{H}), 6.96(\mathrm{~d}, J=8.9 \mathrm{~Hz}, 2 \mathrm{H}), 6.56$ $(\mathrm{d}, J=15.1 \mathrm{~Hz}, 1 \mathrm{H}), 3.83$ (s, $3 \mathrm{H}), 2.32(\mathrm{~s}, 3 \mathrm{H}) ;{ }^{13} \mathrm{C} \mathrm{NMR}\left(125 \mathrm{MHz} \mathrm{CDCl}_{3}\right) \delta$ 163.1, $141.73,141.69,140.7,133.0,130.8,129.32,129.30,129.2,128.2$, 126.5, 126.1, 122.8, 115.6, 55.8, 21.5; IR (thin film) 3042, 2925, 1587, 1489, 1279, 1259, 1137, 1083, 966, $809,746,658 \mathrm{~cm}^{-1}$; HRMS (FAB) calculated for $\mathrm{C}_{22} \mathrm{H}_{21} \mathrm{NO}_{3} \mathrm{~S}_{2}, 412.1041 \mathrm{~m} / z(\mathrm{M}+\mathrm{H})^{+}$; observed 412.1044.

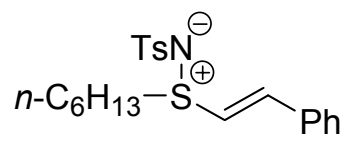

\section{Sulfilimine 5i (Table 1, entry i)}

${ }^{1} \mathrm{H}$ NMR $\left(500 \mathrm{MHz} \mathrm{CDCl}_{3}\right) \delta 7.82(\mathrm{~d}, J=8.1 \mathrm{~Hz}, 2 \mathrm{H}), 7.39-7.36(\mathrm{~m}, 5 \mathrm{H}), 7.24(\mathrm{~d}, J=$ $15.0 \mathrm{~Hz}, 1 \mathrm{H}), 7.20$ (d, $J=8.1 \mathrm{~Hz}, 2 \mathrm{H}), 6.55(\mathrm{~d}, J=15.3 \mathrm{~Hz}, 1 \mathrm{H}), 3.09-2.88(\mathrm{~m}, 2 \mathrm{H})$, 2.33 (s, $3 \mathrm{H}), 1.1 .65-1.59(\mathrm{~m}, 2 \mathrm{H}), 1.34-1.16(\mathrm{~m}, 6 \mathrm{H}), 0.86(\mathrm{t}, J=7.0 \mathrm{~Hz}, 3 \mathrm{H}) ;{ }^{13} \mathrm{C}$ NMR (125 MHz $\left.\mathrm{CDCl}_{3}\right) \delta 142.4,142.0,141.7,133.1,131.0,129.5,129.3,128.2,126.6$, 121.0, 51.4, 31.4, 28.1, 23.0, 22.5, 21.6, 14.2; IR (thin film) 2925, 2856, 1450, 1279, 1141, 1083, 971, 810, 731, $658 \mathrm{~cm}^{-1}$; HRMS (FAB) calculated for $\mathrm{C}_{21} \mathrm{H}_{27} \mathrm{NO}_{2} \mathrm{~S}_{2}$, $390.1561 \mathrm{~m} / \mathrm{z}(\mathrm{M}+\mathrm{H})^{+}$; observed 390.1584 . 


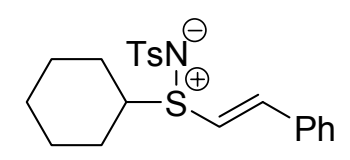

\section{Sulfilimine 5j (Table 1, entry j)}

${ }^{1} \mathrm{H}$ NMR (500 MHz CDCl $) \delta 7.78(\mathrm{~d}, J=8.2 \mathrm{~Hz}, 2 \mathrm{H}), 7.36-7.33(\mathrm{~m}, 5 \mathrm{H}), 7.16(\mathrm{~d}, J=$ $8.0 \mathrm{~Hz}, 2 \mathrm{H}), 7.13$ (d, $J=15.3 \mathrm{~Hz}, 1 \mathrm{H}), 6.50(\mathrm{~d}, J=15.3 \mathrm{~Hz}, 1 \mathrm{H}), 3.04-2.98(\mathrm{~m}, 1 \mathrm{H})$, 2.11-2.08 (m, 2 H), 2.29 (s, 3 H), 2.05-2.03 (m, 1 H), 1.95-1.93 (m, 1 H), 1.81-1.79 (m, 2 H), 1.64-1.61 (m, $1 \mathrm{H}), 1.51-1.17(\mathrm{~m}, 5 \mathrm{H}) ;{ }^{13} \mathrm{C} \mathrm{NMR}\left(125 \mathrm{MHz} \mathrm{CDCl}_{3}\right) \delta$ 143.0, 141.7, 141.6, 133.1, 130.7, 129.2, 129.1, 128.0, 126.4, 119.0, 105.0, 60.1, 26.8, 25.7, 25.3, 25.2, 25.1, 21.4; IR (thin film) 3030, 2926, 2854, 1597, 1446, 1275, 1140, 1084, 969, 741, 652, $569,549 \mathrm{~cm}^{-1}$.

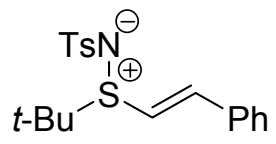

\section{Sulfilimine 5k (Table 1, entry k)}

${ }^{1} \mathrm{H}$ NMR (500 MHz CDCl $) \delta 7.79(\mathrm{~d}, J=8.2 \mathrm{~Hz}, 2 \mathrm{H}), 7.40-7.34(\mathrm{~m}, 5 \mathrm{H}), 7.19$ (d, $J=$ $15.3 \mathrm{~Hz}, 1 \mathrm{H}), 7.17$ (d, $J=8.0 \mathrm{~Hz}, 2 \mathrm{H}), 6.46$ (d, $J=15.2 \mathrm{~Hz}, 1 \mathrm{H}), 2.31$ (s, $3 \mathrm{H}), 1.32$ (s, $9 \mathrm{H}) ;{ }^{13} \mathrm{C}$ NMR $\left(125 \mathrm{MHz} \mathrm{CDCl}_{3}\right) \delta 143.5,141.5,133.5,130.7,129.8,129.19,129.16$, 128.0, 126.6, 117.5, 58.7, 24.1, 21.4; IR (KBr) 3012, 1288, 1275, 1133, 1080, $966 \mathrm{~cm}^{-1}$; HRMS (FAB) calculated for $\mathrm{C}_{19} \mathrm{H}_{23} \mathrm{NO}_{2} \mathrm{~S}_{2}, 362.1170 \mathrm{~m} / z(\mathrm{M}+\mathrm{H})^{+}$; observed 362.1133 .

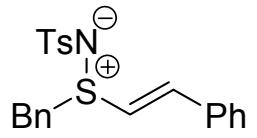

\section{Sulfilimine 5l (Table 1, entry l)}

${ }^{1} \mathrm{H}$ NMR $\left(500 \mathrm{MHz} \mathrm{CDCl}_{3}\right) \delta 7.63(\mathrm{~d}, J=8.2 \mathrm{~Hz}, 2 \mathrm{H}), 7.35-7.18(\mathrm{~m}, 10 \mathrm{H}), 7.07(\mathrm{~d}, J=$ $14.9 \mathrm{~Hz}, 1 \mathrm{H}), 7.06$ (d, $J=8.8 \mathrm{~Hz}, 2 \mathrm{H}), 6.48$ (d, $J=15.3 \mathrm{~Hz}, 1 \mathrm{H}), 4.28$ (d, $J=12.5 \mathrm{~Hz}$, $1 \mathrm{H}), 4.19(\mathrm{~d}, J=12.5 \mathrm{~Hz}, 1 \mathrm{H}), 2.27(\mathrm{~s}, 3 \mathrm{H}) ;{ }^{13} \mathrm{C} \mathrm{NMR}\left(125 \mathrm{MHz} \mathrm{CDCl}_{3}\right) \delta 142.7$, 141.7, 141.5, 133.0, 130.9, 129.5, 129.4, 129.25, 129.19, 128.1, 127.8, 126.4, 120.0, 58.1, 21.5; IR (KBr) 3027, 2977, 1280, 1137, 1085, 959, 897, 822, 743, 703, 650, 570, 550 $\mathrm{cm}^{-1}$; HRMS (FAB) calculated for $\mathrm{C}_{22} \mathrm{H}_{21} \mathrm{NO}_{2} \mathrm{~S}_{2}, 396.1092 \mathrm{~m} / \mathrm{z}(\mathrm{M}+\mathrm{H})^{+}$; observed 396.1096 . 


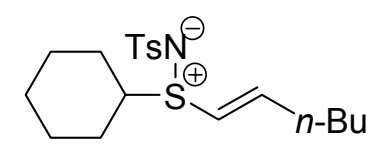

\section{Sulfilimine 8 (Table 2, entry 1)}

${ }^{1} \mathrm{H}$ NMR (500 MHz CDCl 3 ) $\delta 7.74(\mathrm{~d}, J=8.2 \mathrm{~Hz}, 2 \mathrm{H}), 7.19(\mathrm{~d}, J=8.2 \mathrm{~Hz}, 2 \mathrm{H}), 6.42$ (dt, $J=14.9,7.0 \mathrm{~Hz}, 1 \mathrm{H}), 5.89$ (d, $J=15.2 \mathrm{~Hz}, 1 \mathrm{H}), 2.89-2.85$ (m, $1 \mathrm{H}), 2.36$ (s, $3 \mathrm{H}), 2.16-$ 2.12 (m, 2 H), 2.04-2.02 (m, 1 H), 1.90-1.79 (m, 2 H), 1.65-1.63 (m, 1 H), 1.42-1.19 (m, $10 \mathrm{H}), 0.87(\mathrm{t}, J=7.2 \mathrm{~Hz}, 3 \mathrm{H}) ;{ }^{13} \mathrm{C} \mathrm{NMR}\left(125 \mathrm{MHz} \mathrm{CDCl}_{3}\right) \delta 148.1,141.9,141.5,129.2$, $126.5,121.2,59.4,32.3,30.0,26.6,25.8,25.3,25.2,25.0,22.2,21.5,13.9$; IR (thin film) 2925, 2860, 1735, 1452, 1279, 1142, 1088, 976, 814, 731, $653 \mathrm{~cm}^{-1}$; HRMS (FAB) calculated for $\mathrm{C}_{19} \mathrm{H}_{29} \mathrm{NO}_{2} \mathrm{~S}_{2}, 368.1718 \mathrm{~m} / z(\mathrm{M}+\mathrm{H})^{+}$; observed 368.1725.

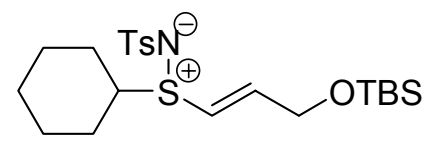

\section{Sulfilimine 10 (Table 2, entry 2)}

${ }^{1} \mathrm{H}$ NMR (500 MHz $\left.\mathrm{CDCl}_{3}\right) \delta 7.72(\mathrm{~d}, J=8.2 \mathrm{~Hz}, 2 \mathrm{H}), 7.17(\mathrm{~d}, J=8.0 \mathrm{~Hz}, 2 \mathrm{H}), 6.56(\mathrm{dt}$, $J=14.5,2.9 \mathrm{~Hz}, 1 \mathrm{H}), 6.16(\mathrm{dt}, J=14.5,2.2 \mathrm{~Hz}, 1 \mathrm{H}), 4.22(\mathrm{t}, J=2.7 \mathrm{~Hz}, 2 \mathrm{H}), 2.87-2.80$ $(\mathrm{m}, 1 \mathrm{H}), 2.34(\mathrm{~s}, 3 \mathrm{H}), 2.01-1.15(\mathrm{~m}, 10 \mathrm{H}), 0.86(\mathrm{~s}, 9 \mathrm{H}), 0.01(\mathrm{~s}, 6 \mathrm{H}) ;{ }^{13} \mathrm{C} \mathrm{NMR}(125$ $\left.\mathrm{MHz} \mathrm{CDCl}_{3}\right) \delta 145.2,142.2,141.3,129.1,126.5,120.2$, 77.6, 62.3, 60.0, 26.7, 25.9, 25.7, 25.3, 25.1, 21.4, 18.3, -5.0; IR (thin film) 2930, 2857, 1599, 1453, 1296, 1281, 1142, $1089,964,838,814,657 \mathrm{~cm}^{-1}$; HRMS (FAB) calculated for $\mathrm{C}_{22} \mathrm{H}_{37} \mathrm{NO}_{3} \mathrm{~S}_{2} \mathrm{Si}, 456.2062$ $m / z(\mathrm{M}+\mathrm{H})^{+}$; observed 456.2055.

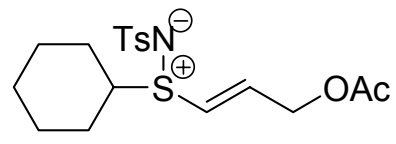

\section{Sulfilimine 12 (Table 2, entry 3)}

${ }^{1} \mathrm{H}$ NMR (500 MHz CDCl 3$) \delta 7.67(\mathrm{~d}, J=8.2 \mathrm{~Hz}, 2 \mathrm{H}), 7.15(\mathrm{~d}, J=8.1 \mathrm{~Hz}, 2 \mathrm{H}), 6.47$ (dt, $J=14.8,4.3 \mathrm{~Hz}, 1 \mathrm{H}$ ), 6.13 (app triplet, $J=15.5,14.9 \mathrm{~Hz}, 1 \mathrm{H}), 4.59$ (d, $J=2.8 \mathrm{~Hz}, 2 \mathrm{H}$ ), 2.87-2.80 (m, $1 \mathrm{H}), 2.31(\mathrm{~s}, 3 \mathrm{H}), 2.02(\mathrm{~s}, 3 \mathrm{H}), 1.86-1.13(\mathrm{~m}, 10 \mathrm{H}) ;{ }^{13} \mathrm{C} \mathrm{NMR}(125 \mathrm{MHz}$ $\left.\mathrm{CDCl}_{3}\right) \delta 170.0,141.6,141.3,138.7,129.1,126.2,122.3,62.2,59.3,26.6,26.3,25.8$, 25.1, 24.9, 24.87, 21.4; IR (thin film) 2935, 2858, 1747, 1451, 1280, 1229, 1143, 1089, 973, 915, 733, $656 \mathrm{~cm}^{-1}$; HRMS (FAB) calculated for $\mathrm{C}_{18} \mathrm{H}_{25} \mathrm{NO}_{4} \mathrm{~S}_{2}, 384.1225 \mathrm{~m} / z(\mathrm{M}+$ $\mathrm{H})^{+}$; observed 384.1266 . 


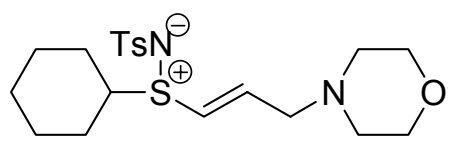

\section{Sulfilimine 14 (Table 2, entry 4)}

${ }^{1} \mathrm{H}$ NMR (500 MHz CDCl 3$) \delta 7.71(\mathrm{~d}, J=8.2 \mathrm{~Hz}, 2 \mathrm{H}), 7.17(\mathrm{~d}, J=8.0 \mathrm{~Hz}, 2 \mathrm{H}), 6.43$ (dt, $J=15.0,5.7 \mathrm{~Hz}, 1 \mathrm{H}), 6.13(\mathrm{dt}, J=15.0,2.5 \mathrm{~Hz}, 1 \mathrm{H}), 3.61(\mathrm{t}, J=4.6 \mathrm{~Hz}, 4 \mathrm{H}), 3.03$ (dd, $J=5.6,2.5 \mathrm{~Hz}, 2 \mathrm{H}), 2.90-2.83(\mathrm{~m}, 1 \mathrm{H}), 2.33(\mathrm{~s}, 3 \mathrm{H}), 2.27$ (t, $J=4.6 \mathrm{~Hz}, 4 \mathrm{H}), 1.98-$ $1.15(\mathrm{~m}, 10 \mathrm{H}) ;{ }^{13} \mathrm{C} \mathrm{NMR}\left(125 \mathrm{MHz} \mathrm{CDCl}_{3}\right) \delta 142.9,142.1,141.3,129.0,126.4,123.7$, $66.8,59.6,59.1,53.5,26.7,25.6,25.1,24.9,21.3,14.1$; IR (thin film) 2933, 2856, 2811, 1453, 1349, 1296, 1280, 1143, 1117, 1089, 972, 867, 816, 734, 657, 575, $552 \mathrm{~cm}^{-1}$; HRMS (FAB) calculated for $\mathrm{C}_{20} \mathrm{H}_{30} \mathrm{~N}_{2} \mathrm{O}_{3} \mathrm{~S}_{2}, 411.1776 \mathrm{~m} / z(\mathrm{M}+\mathrm{H})^{+}$; observed 411.1793.

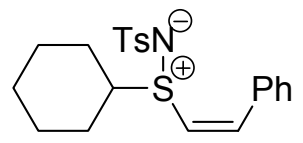

\section{Sulfilimine 16 (Table 2, entry 5)}

${ }^{1} \mathrm{H}$ NMR $\left(500 \mathrm{MHz} \mathrm{CDCl}_{3}\right) \delta 7.66(\mathrm{~d}, J=8.2 \mathrm{~Hz}, 2 \mathrm{H}), 7.36-7.29$ (m, $\left.5 \mathrm{H}\right), 7.13(\mathrm{~d}, J=$ $10.4 \mathrm{~Hz}, 1 \mathrm{H}), 7.06(\mathrm{~d}, J=8.1 \mathrm{~Hz}, 2 \mathrm{H}), 6.10$ (d, $J=10.4 \mathrm{~Hz}, 1 \mathrm{H}), 3.01-2.95(\mathrm{~m}, 1 \mathrm{H})$, 2.29 (s, 3 H), 2.15-2.12 (m, 1 H), 1.90-1.87 (m, 1 H), 1.80-1.76 (m, 1 H), 1.69-1.67 (m, 1 $\mathrm{H}), 1.61-1.58(\mathrm{~m}, 1 \mathrm{H}), 1.34-1.17(\mathrm{~m}, 4 \mathrm{H}) ;{ }^{13} \mathrm{C} \mathrm{NMR}\left(125 \mathrm{MHz} \mathrm{CDCl}_{3}\right) \delta 143.4,141.6$, $141.4,132.8,130.0,129.5,129.1,128.8,126.4,123.3,60.7,26.4,26.1,25.2,25.1,24.9$, 21.4; IR (thin film) 3029, 2927, 2855, 1598, 1491, 1445, 1281, 1137, 1086, 958, 779, 686, $651 \mathrm{~cm}^{-1}$.

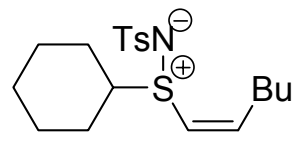

\section{Sulfilimine 18 (Table 2, entry 6)}

${ }^{1} \mathrm{H}$ NMR (500 MHz CDCl 3$) \delta 7.74(\mathrm{~d}, J=8.2 \mathrm{~Hz}, 2 \mathrm{H}), 7.18(\mathrm{~d}, J=8.4 \mathrm{~Hz}, 2 \mathrm{H}), 6.27$ (dt, $J=9.3,7.8 \mathrm{~Hz}, 1 \mathrm{H}), 5.93(\mathrm{~d}, J=9.3 \mathrm{~Hz}, 1 \mathrm{H}), 2.95-2.87(\mathrm{~m}, 1 \mathrm{H}), 2.35$ (s, $3 \mathrm{H}), 2.22-$ $1.18(\mathrm{~m}, 16 \mathrm{H}), 0.85$ (t, $J=6.6 \mathrm{~Hz}, 3 \mathrm{H}) ;{ }^{13} \mathrm{C} \mathrm{NMR}\left(125 \mathrm{MHz} \mathrm{CDCl}_{3}\right) \delta 147.8,142.0$, $141.4,129.1$, 126.5, 123.7, 59.8, 30.6, 29.9, 29.8, 26.3, 25.3, 25.1, 24.8, 22.2 , 21.5, 13.9; IR (thin film) 2929, 2856, 1452, 1281, 1142, 1089, $965 \mathrm{~cm}^{-1}$. 


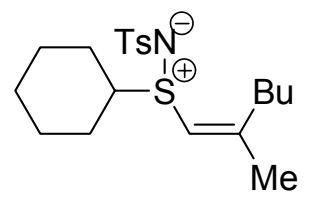

\section{Sulfilimine 20 (Table 2, entry 7)}

${ }^{1} \mathrm{H}$ NMR (500 MHz $\left.\mathrm{CDCl}_{3}\right) \delta 7.74(\mathrm{~d}, J=8.2 \mathrm{~Hz}, 2 \mathrm{H}), 7.17(\mathrm{~d}, J=8.1 \mathrm{~Hz}, 2 \mathrm{H}), 5.70(\mathrm{~s}$, $1 \mathrm{H}), 2.90-2.88(\mathrm{~m}, 1 \mathrm{H}), 2.36$ (s, $3 \mathrm{H}), 2.25-2.10$ (m, 3 H), 1.86-1.62 (m, 7 H), 1.40-1.21 $(\mathrm{m}, 9 \mathrm{H}), 0.86(\mathrm{t}, J=7.0 \mathrm{~Hz}, 3 \mathrm{H}) ;{ }^{13} \mathrm{C} \mathrm{NMR}\left(125 \mathrm{MHz}^{\mathrm{CDCl}}{ }_{3}\right) \delta 158.6,142.1,141.3$, $129.1,126.6,118.5,59.8,34.3,29.9,26.5,26.4,25.3,25.2,24.9,23.4,22.6,21.5,14.0$; IR (thin film) 2931, 2858, 1452, 1294, 1280, 1141, 1089, 966, 815, 755, $654 \mathrm{~cm}^{-1}$; HRMS (FAB) calculated for $\mathrm{C}_{20} \mathrm{H}_{31} \mathrm{NO}_{2} \mathrm{~S}_{2}, 382.1874 \mathrm{~m} / z(\mathrm{M}+\mathrm{H})^{+}$; observed 382.1855.

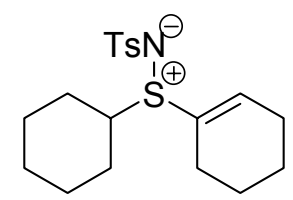

\section{Sulfilimine 22 (Table 2, entry 8)}

${ }^{1} \mathrm{H}$ NMR (500 MHz $\left.\mathrm{CDCl}_{3}\right) \delta 7.72(\mathrm{~d}, J=8.2 \mathrm{~Hz}, 2 \mathrm{H}), 7.19(\mathrm{~d}, J=8.0 \mathrm{~Hz}, 2 \mathrm{H}), 6.40$ 6.39 (m, $1 \mathrm{H}), 2.98-2.94(\mathrm{~m}, 1 \mathrm{H}), 2.45-2.41$ (m, $1 \mathrm{H}), 2.36$ (s, $3 \mathrm{H}), 2.17-1.21(\mathrm{~m}, 17 \mathrm{H})$;

${ }^{13} \mathrm{C}$ NMR $\left(125 \mathrm{MHz} \mathrm{CDCl}_{3}\right) \delta 141.9,141.3,141.2,131.7,129.1,126.5,54.9,27.1,26.4$, 26.0, 25.3, 25.1, 24.8, 22.1, 21.5, 21.46, 20.6; IR (thin film) 2932, 2858, 1450, 1281, 1142, 1089, 969, 815, 762, 734; HRMS (FAB) calculated for $\mathrm{C}_{19} \mathrm{H}_{27} \mathrm{NO}_{2} \mathrm{~S}_{2}, 366.1561 \mathrm{~m} / \mathrm{z}$ $(\mathrm{M}+\mathrm{H})^{+}$; observed 366.1570 .

\section{References:}

1) Marino, J. P. ; Rubio, M. B. ; Cao, G.; de Dios, A. J. Am. Chem. Soc. 2002, 124, 13398.

2) Campbell, M. M.; Johnson, G. Chem. Rev. 1978, 78, 65.

3) Rudolph, J.; Sennhenn, P. C.; Vlaar, C. P.; Sharpless, K. B. Angew. Chem., Int. Ed. 1996, 35, 2810.

4) Marzinzik, A. L.; Sharpless, K. B. J. Org. Chem. 2001, 66, 594.

5) Danheiser, R. L.; Savariar, S. Tetrahedron Lett. 1987, 28, 3299.

6) Trost, B. M.; Sudhakar, A. R. J. Am. Chem. Soc. 1987, 109, 3792. 